\title{
四苯基卟㕲 $\left(\mathrm{H}_{2}\right.$ TPP $)$ 与铕 (III)-四苯基 叶啉 $(\mathrm{Eu}-\mathrm{TPP})$ 在丙酮介质中的电还 原行为一一溶解氧的影响
}

黄云辉 关福玉 高小霞 (北京大学化学系,北京 100871)

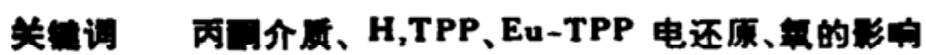

水溶液中稀土离子不容易与水溶性卟晽如 TPPS, ${ }^{\text {[1] }}$ 形成络合物,一般要在非水介质中先 行合成. 本文在丙阙介质中（含 $0.1 \mathrm{~mol} / \mathrm{LTEAP}$ ) 有 $\mathrm{H}_{2} \mathrm{TPP}$ 存在下加人 $\mathrm{Eu}\left(\mathrm{ClO}_{4}\right)_{3}$, 立即 得到 $1: 1$ 络合物. 伏安图上络合物还原峰电位在 $-0.28 \mathrm{~V}$ (vs. $\mathrm{Ag} / \mathrm{AgCl}$ ) 是其中 $\mathrm{Eu}^{3+}$ 还原 到 $\mathrm{Eu}^{2+}$, 峰高与 $\mathrm{Eu}^{3+}$ 浓度 $5 \times 10^{-6}-5 \times 10^{-5} \mathrm{~mol} / \mathrm{L}$ 间有良好线性关系, 不受溶解篻的 影响. 但若电位扫描至 $-1.2 \mathrm{~V}$ 后再作第二次循环伏安时, 因为溶解 $\mathrm{O}_{2}^{-}$在 $-0.8 \mathrm{~V}$ 附近还原 为超氧基 $\mathrm{O}_{2}^{-}$, 使得络合物波降低、变形, 并在 $-0.7 \mathrm{~V}$ 出现一个 $\mathrm{Eu}^{3+}-\mathrm{TPP} \cdot \mathrm{O}_{2}^{2-*}$ 的还原蜂.

\section{一、实验部分}

1. 仪哭美国 PAR174A 极谱仪, M303 静承滴电极, 参比电极用双盐桥与丙酮连通, 电位均相对于 $\mathrm{Ag} / \mathrm{AgCl}, \mathrm{KCl}$ (aq.).

2. 试驻丙酮, 分析纯 (北京化工厂), 除水方法同文献 [2]. 高氯酸季铵盐 $\left(\mathrm{C}_{2} \mathrm{H}_{5}\right)$, $\mathrm{NClO}_{4}$ (TEAP) 按文献 [3] 制得. 四苯基卟啉 $\mathrm{H}_{2} \mathrm{TPP}$ (日本同仁化学研究所), $\mathrm{C}_{44} \mathrm{H}_{30} \mathrm{~N}_{4}$, 分 子盟 614.75 , 称取一定量在丙酮中迴流溶解后, 配成 $1.0 \times 10^{-4} \mathrm{~mol} / \mathrm{L}$ 溶液. $\mathrm{Eu}_{2} \mathrm{O}_{3}$ (北京大 学化学系制, 纯度为 $99.9 \%$ ) 溶于稀 $\mathrm{HClO}_{4}$, 蒸发近干, 在 $120^{\circ} \mathrm{C}$ 真空干燥 $48 \mathrm{~h}$ 后, 配成 $1.0 \times 10^{-2} \mathrm{~mol} / \mathrm{L}$ 丙酮溶液, 高纯氮除氧 $4 \mathrm{~min}$.

\section{二、结果与论论}

先观察四苯基卟啉 $\left(\mathrm{H}_{2} \mathrm{TPP}\right)$ 在丙酮中的电还原行为, 然后加人 $\mathrm{Eu}\left(\mathrm{ClO}_{4}\right)_{3}$ 直接形成络 合物,并研究溶解革对络合物还原波的影响.

1. $\mathrm{H}_{2}$ TPP 在丙缃中的伏安行为图 1 是 $\mathrm{H}_{2}$ TPP 在丙酮(含 $0.1 \mathrm{~mol} / \mathrm{L}$ TEAP) 中的 很环伏安图. 其中 $b$ 有 5 个峰, 最前的 $\mathrm{P}_{0}\left(\mathrm{P}_{0}^{\prime}\right)$ 尖峰 $(-0.82 \mathrm{~V})$ 是丙酮中难以除尽的 $\mathrm{O}_{2}$ 与 $\mathrm{H}_{2} \mathrm{TPP}$ 键合的还原峰. 文献 [4] 中称为前波, 未作解释. 本文中证明是 $\mathrm{H}_{2} \mathrm{TPP} \cdot \mathrm{O}_{2}$ 还原为 $\mathrm{H}_{2} \mathrm{TPPO}_{2}^{-}$的波. 图 1-b 中其后有四步波, $\mathrm{P}_{1}(-1.02 \mathrm{~V})$ 和 $\mathrm{P}_{2}(-1.42 \mathrm{~V})$ 是两对近乎可逆 的波峰, 它们的阴阳极峰电位之差均约 $60 \mathrm{mV}$, 故认为是 $1 \mathrm{e}^{-}$的电极过程. $\mathrm{P}_{3}(-1.99 \mathrm{~V})$

1991-09-05 收稿, 1992-03-11 收修改穗

* 国家臽然科学基金资助项目 
和 $\mathbf{P}_{4}(-2.14 \mathrm{~V})$ 是各得 $2 \mathrm{e}^{-}$的不可逆过程. 从直流极谱极限扩散电流 ${ }^{i d}$ 的半对数分析大 致可以证明, 数值列于表 1. 这些波都是卟啉环上的分步还原, 共得 $6 \mathrm{e}^{-}$, 与水溶液中 $P_{1}$ 为 $2 \mathrm{e}^{-}, \mathrm{P}_{2} 、 \mathrm{P}_{3}$ 各为 $2 \mathrm{e}^{-}$, 共 $6 \mathrm{e}^{-}$还原基本相同.

表 $1 \quad H_{2}$ TPP 还原波的半对数分析

\begin{tabular}{|c|c|c|c|}
\hline 还原波 & $E_{y_{2}}(V)$ & $\begin{array}{c}\text { 平均极限扩散电流 } \\
\underbrace{}_{d}(\mu \mathrm{\Lambda})\end{array}$ & $\begin{array}{c}E \sim \lg \frac{\bar{i}}{i, \bar{i}}, \\
\text { 料事 }(\mathrm{mV})\end{array}$ \\
\hline$P_{1}$ & -0.99 & 0.213 & 60.5 \\
\hline $\mathbf{P}_{2}$ & -1.37 & 0.250 & 66.9 \\
\hline$P_{3}$ & -1.90 & 0.485 & 45.8 \\
\hline P. & -2.07 & 0.455 & 43.4 \\
\hline
\end{tabular}

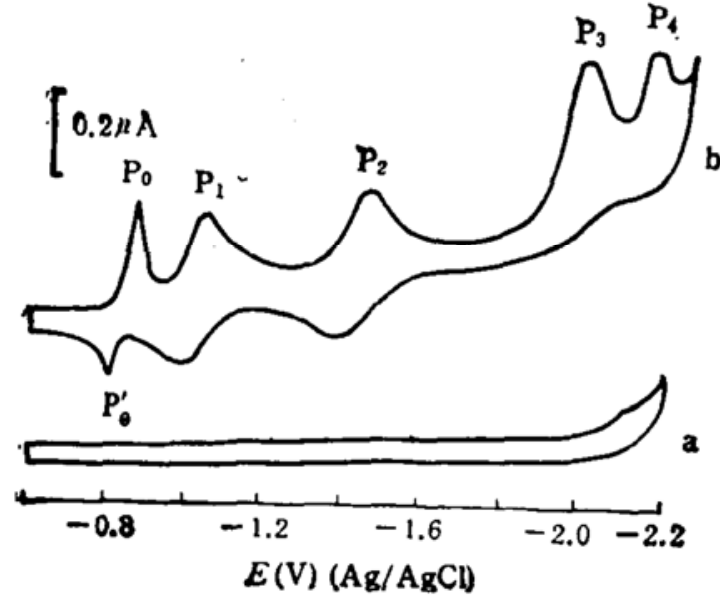

图 1 H, TPP、循环伏安图

s. 丙整 $+0.1 \mathrm{~mol} / \mathrm{LTEAP}$;

b. $\mathrm{a}+5.0 \times 10^{-3} \mathrm{~mol} / \mathrm{LH}_{2} \mathrm{TPP}$.

通纯 $\mathrm{N}_{2}$ 除 $\mathrm{O}_{2}, \nu=200 \mathrm{mV} / \mathrm{s}$

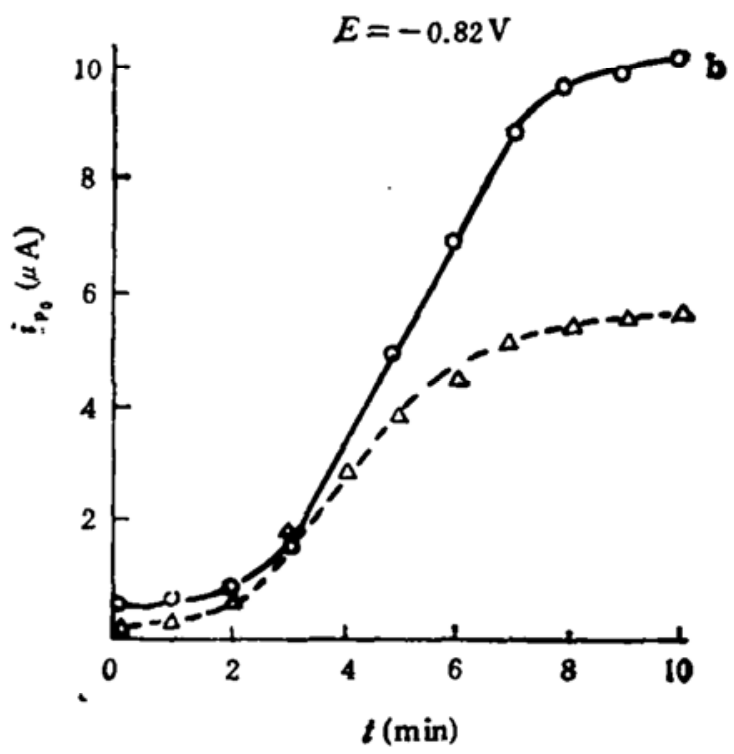

图 2 溶解氧的影响

.. 丙期 $+0.1 \mathrm{~mol} / \mathrm{L}$ TEAP;

b. $+5.0 \times 10^{-1} \mathrm{~mol} / \mathrm{LH}_{2} \mathrm{TPP}$.

通 $\mathrm{N}_{2}$ 除 $\mathrm{O}_{2} 4 \mathrm{~min}$, 效㫪不同时间 (

在图 1-b 的溶液中若加人 $0.5-1.0 \% \mathrm{H}_{2} \mathrm{O}, \mathrm{P}_{0}$ 峰降低, $\mathrm{P}_{0}^{\prime}$ 峰消失, 而 $\mathrm{P}_{3} 、 \mathrm{P}_{4}$ 蜂电位 均正移约 $0.4 \mathrm{~V}$, 表明 $\mathrm{P}_{0}$ 是末能除尽的 $\mathrm{O}_{2}$ 还原为 $\mathrm{O}_{2}^{-}$, 与 $\mathrm{H}_{2} \mathrm{O}$ 作用消除了 $\mathrm{O}_{2}^{-}$和 $\mathrm{P}_{0}^{\prime}, \mathrm{P}_{3}$ 、 $P_{4}$ 电位正移表明有 $\mathrm{H}^{+}$参加电极反应, 因此 $\mathrm{H}_{2} \mathrm{TPP}$ 的还原历程写为

$$
\begin{aligned}
& \mathrm{H}_{2} \mathrm{TPP}+\mathrm{e}^{-} \stackrel{\mathrm{P}_{1}}{\rightleftarrows} \mathrm{H}_{2} \mathrm{TPP}^{-} \pi \text { 阴离子自由基 } \\
& \mathrm{H}_{2} \mathrm{TPP}^{-}+\mathrm{e}^{-} \stackrel{\mathrm{P}_{2}}{\rightleftarrows} \mathrm{H}_{2} \mathrm{TPP}^{2-} \text { 二阴离子 } \\
& \mathrm{H}_{2} \mathrm{TPP}^{2-}+n \mathrm{H}^{+}+2 \mathrm{e}^{-\stackrel{\mathrm{P}_{3}}{\longrightarrow}} \mathrm{H}_{2} \mathrm{TPPH}_{n}^{(4-m)-} \\
& \mathrm{H}_{2} \mathrm{TPPH}_{n}^{(4-n)-}+m \mathrm{H}^{+}+2 \mathrm{e}^{-\stackrel{\mathrm{P} \leftrightarrow}{\longrightarrow}} \mathrm{H}_{2} \mathrm{TPPH}_{n+m}^{(6-(n+m) 1-}
\end{aligned}
$$

为了进一步证明 $P_{0} 、 P_{0}^{\prime}$ 是 $O_{2}$ 的还原和氧化, 作图 2. 其中 a 是丙酮中 $P_{0}$ 电流随放 时间延长而增加, $2 \mathrm{~min}$ 后增长明显,到 $7 \mathrm{~min}$ 后趋于平稳, 而 $\mathrm{b}$ 是有 $\mathrm{H}_{2} \mathrm{TPP}$ 存在, $2 \mathrm{~min}$ 后 吸人 $\mathrm{O}_{2}$ 更明显, $\mathrm{P}_{0}$ 迅速升高, $7 \mathrm{~min}$ 后也达到饱和, 常温常压下水溶液中溶解 $\mathrm{O}_{2}$ 约为 $1.23 \times 10^{-3} \mathrm{~mol} / \mathrm{L}$, 通 $\mathrm{N}_{2}$ 基本可以除去, 但丙酮中不能出, 有 $\mathrm{H}_{2} \mathrm{TPP}$ 时更不能除尽. 图 2 
证明丙酮中 $\mathrm{H}_{2}$ TPP 很容易吸人空气中 $\mathrm{O}_{2}$, 这是因为 $\mathrm{H}_{2}$ TPP 与 $\mathrm{O}_{2}$ 可能键合成 $\mathrm{H}_{2}$ TPP $\mathrm{O}_{2}$, 在非水介质中可以还原为 $\mathrm{O}_{2}^{-}$而出现 $\mathrm{P}_{0} 、 \mathrm{P}_{0}^{\prime}$ 一对尖峰. 它在稀土卟啉络合物电还原中 有很大影响。

2. 销（III)-TPP 络合物的电还原和重的影响稀土离子在水溶液中难于与水溶性㠴 味络合. 在丙酮介质中当有 $\mathrm{H}_{2} \mathrm{TPP}$ 存在, 加人等廖尔的 $\mathrm{Eu}\left(\mathrm{ClO}_{4}\right)_{3}$, 可以立刻形成络合物, 溶液由红色变为棕色, $\mathrm{H}_{2} \mathrm{TPP}$ 原来吸收光谱的 4 个 $\theta$ 带 $(500-700 \mathrm{~nm})$ 发生明显变 化,并在 $434 \mathrm{~nm}$ 出现一个新吸收峰. 用光度等摩尔变化法求络合物组成比为 $1: 1$, 可能写为 [Eu $\left.\mathrm{u}^{3+}-\mathrm{TPP}\right] \cdot \mathrm{ClO}_{4}^{-} \cdot$ 同时在图 3 上可以看到在 $-0.28 \mathrm{~V}$ 处有一个络合物 $\mathrm{Pc}_{\mathrm{c}}$. 图 3 虚线 是只有 $\mathrm{Eu}\left(\mathrm{ClO}_{4}\right)_{3}$ 时 $\mathrm{Eu}^{3+}$ 的两步还原波. 实线上除了 $\mathrm{P}_{6}$ 外, 仍有 $\mathrm{P}_{0}\left(\mathrm{P}_{0}^{\prime}\right), \mathrm{P}_{1}$ 和 $\mathrm{P}_{3} 、 \mathrm{P}_{4}$, 其 中 $P_{2}$ 不明显, 但有它的氧化峰. $P_{3} 、 P_{4}$ 峰电位比图 1 中的有所正移. 络合物 $P_{0}$ 峰相当稳 定, 溶液在室温放置 $8 \mathrm{~h}$, 峰高基本不变, 峰电位略有负移是吸收了空气中水分的缘故. 测峰 高与 $\mathrm{Eu}^{3+}$ 浓度在 $5.0 \times 10^{-6}-7 \times 10^{-3} \mathrm{~mol} / \mathrm{L}$ 间成正比, 直线通过原点, 因为 $\mathrm{P}_{6}$ 峰在 $\mathrm{O}_{2}$ 还原之前而不受氧的影响.

但是, 电位扫描到不同电位后回扫,作第二次循环伏安时情况就大不一样. 图 4 上a是扫 描到 $-0.5 \mathrm{~V}$ 便回扫, 第二次循环时(虚线) $P_{c}$ 峰稍有降低. $b$ 是扫到 $-0.9 \mathrm{~V}$ 后再第二次扫 描, 除 $P_{0}$ 峰降低外还在 $-0.70 \mathrm{~V}$ 出现一新蜂 $P_{N}$ 和较小的 $P_{0}\left(P_{0}^{\prime}\right)$ 尖峰。 $c$ 是扫描到 -1.2 $V$ 后再二次循环, 虚线示出 $P_{0}$ 峰变形且 $P_{N}$ 峰增高、变宽, 使 $P_{0}$ 和 $P_{0}^{\prime}$ 消失, 紧接后面 $P_{1}$ 峰. 可以看到 $P_{6}$ 峰在第一次扫描时都能保持不变,但电位扫描超过 $-0.8 \mathrm{~V}$ 回扫并作二次秫环 时 $\mathrm{P}_{0}$ 峰变为 $\mathrm{P}_{\mathrm{N}}$ 峰. 说明 $\mathrm{O}_{2}$ 的还原影响了络合物组成. 文献报道 $\mathrm{Co}$ (II), $\mathrm{Fe}$ (II) 吓啉 络合物可用作研究动物体内血红蛋白、肌红蛋白等载氧体的模型物. 金属卟啉中处于内配位

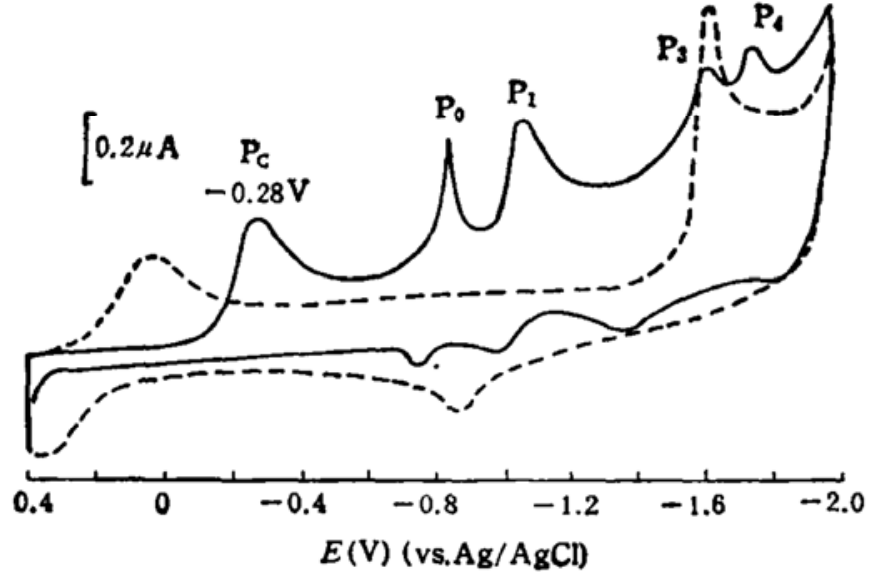

图 $3 \mathrm{Eu}^{\mathrm{s}+}$ 和 $\mathrm{Eu}^{\mathrm{s}+}-\mathrm{TPP}$ 的循环伏安图

-.- 为丙露 $+0.1 \mathrm{~mol} / \mathrm{L} \mathrm{TEAP}+5 \times 10^{-3} \mathrm{~mol} / \mathrm{L} \mathrm{Eu}\left(\mathrm{ClO}_{4}\right)_{3}$; 为同虚线条件加 $5 \times 10^{-4} \mathrm{~mol} / \mathrm{L} \mathrm{H}_{2} \mathrm{TPP}$, 通 $\mathrm{N}_{2} 4 \mathrm{~min}$, ,$-200 \mathrm{mV} / \mathrm{s}$

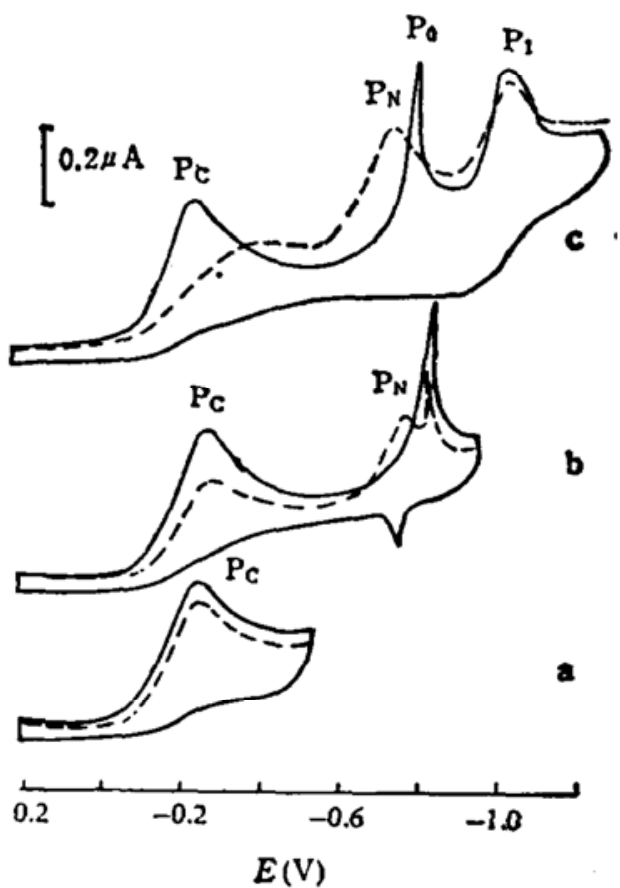

图 4 不同电位的循环伏安图 一一为第一次循环; ----为第二次结环. 丙墽 $+0.1 \mathrm{~mol} / \mathrm{L}$ TEAP; $5.0 \times 10^{-5} \mathrm{~mol} / \mathrm{L}$ $\mathrm{Eu}\left(\mathrm{ClO}_{4}\right)_{3}+5.0 \times 10^{-1} \mathrm{~mol} / \mathrm{L} \mathrm{H} \mathrm{H}_{2}$ TPP. A. $-0.5 \mathrm{~V}$ 回扫; b. $-0.9 \mathrm{~V}$ 回扫; c. $-1.2 \mathrm{~V}$ 回扫, $v=200 \mathrm{mV} / \mathrm{s}$ 
空间的叶啉部分与金属离子的键合力比卟啉环上下轴向部分与金属离子的键合力要强得多. 氧可能以 $\mathrm{O}_{2} 、 \mathrm{O}_{2}^{-}$或 $\mathrm{O}_{2}^{2-}$ 的形式进入金属卟啉的轴向部位键合或取代原有基团, 也可以可逆 地脱离金属卟啉. 这类加合物如 $\left[\mathrm{Zn}(\mathrm{II})\left(\mathrm{TPP}\left(\mathrm{O}_{2}^{-}\right)\right] 、\left[\mathrm{Cr}(\mathrm{III})(\mathrm{TPP})(\mathrm{Py})\left(\mathrm{O}_{2}^{-}\right)\right] 、[\mathrm{Co}\right.$ (III) (TPP) (base) $\left.\left(\mathrm{O}_{2}^{-}\right)\right]$和 $\left[\mathrm{Mn}(\mathrm{IV})(\mathrm{TPP})\left(\mathrm{O}_{2}^{2-}\right)\right]^{[6-9]}$ 等已有报道. 在文献 [5] 中根据桸土能 生成 $\mathrm{RE}\left(\mathrm{O}_{2}^{-}\right)_{3}$ 或 $\mathrm{RE}_{2}\left(\mathrm{O}_{2}^{2-}\right)_{3}$ 用来解释丙酮中由于 $\mathrm{RE}^{3+}$ 与 $\mathrm{O}_{2}^{-}$形成过氧化物薄膜于承电极 导致还原波的分裂，因此对图 4 中的 $P_{c} 、 P_{N} 、 P_{O}$ 的电极过程作下列描述:

$$
\begin{array}{ll}
\mathrm{Eu}^{3+}-\mathrm{TPP}+\mathrm{e}^{-} \rightarrow \mathrm{Eu}^{2+}-\mathrm{TPP} & E_{\mathrm{p}_{\mathrm{c}}}-0.28 \mathrm{~V} \\
\mathrm{O}_{2}+\mathrm{e}^{-} \rightarrow \mathrm{O}_{2}^{-} & \\
\left(\mathrm{H}_{2} \mathrm{TPP} \cdot \mathrm{O}_{2}+\mathrm{e}^{-} \rightarrow \mathrm{H}_{2} \mathrm{TPP} \cdot \mathrm{O}_{2}^{-}\right) & E_{\mathrm{p}_{0}}--0.82 \mathrm{~V} \\
\mathrm{Eu}^{2+} \mathrm{TPP}+\mathrm{O}_{2}^{-} \rightarrow \mathrm{Eu}^{3+}-\mathrm{TPP} \cdot \mathrm{O}_{2}^{2-} & \text { 加合反应 } \\
\mathrm{Eu}^{3+}-\mathrm{TPP} \cdot \mathrm{O}_{2}^{2-}+\mathrm{e}^{-} \rightarrow \mathrm{Eu}^{2+}-\mathrm{TPP} \cdot \mathrm{O}_{2}^{2-}, E_{\mathrm{P}_{\mathrm{N}}}-0.70 \mathrm{~V}
\end{array}
$$

这样 $\mathrm{P}_{\mathrm{N}}$ 峰仍是加合物中 $\mathrm{Eu}^{3+}$ 还原为 $\mathrm{Eu}^{2+}$, 在更负电位才有 TPP 环上的还原.

\section{三、结论}

$\mathrm{H}_{2}$ TPP 在丙酮中有 4 步共 $6 \mathrm{e}^{-}$的还原波和在 $-0.8 \mathrm{~V}$ 附近的一个溶解 $\mathrm{O}_{2}$ 还原为 $\mathrm{O}_{2}^{-}$的 前波。

在丙酮中 $\mathrm{Eu}\left(\mathrm{ClO}_{4}\right)_{3}$ 可以立即与 $\mathrm{H}_{2} \mathrm{TPP}$ 形成 $1: 1$ 络合物, 还原峰 $\left(E_{p_{\mathrm{c}}}--0.28 \mathrm{~V}\right)$ 高 与 $\mathrm{Eu}^{3+}$ 浓度成正比,不受 $\mathrm{O}_{2}$ 的影响.

若电位扫描超过一 $0.8 \mathrm{~V}$ 再作第二次扫描, 有一新峰出现, 可能是 $\mathrm{Eu}^{3+}-\mathrm{TPP} \cdot \mathrm{O}_{2}^{2-}$ 加合 物的还原.

\section{参考文 哭}

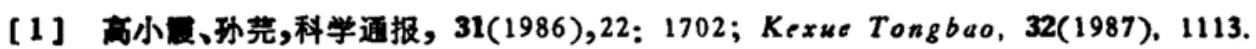

[2] 关福玉、高小而，科学通报，34(1989),22: 1704; Chinese Science Bulletin. 35(1990), 830.

[3] Coksl, E.J., Wise, E.N., J.Electroanal. Chem., 11(1966). 406.

[4] Felton, R.H., Linschtz, H., J.Am. Chem. Soc., 88(1966), 113.

[5] 关褔玉、高小贯,中国稀土学报, 8(1990),2: 166; J. of Chinese Rare Earth Society, 8(1990), 3:212.

[6] Kahai, P.H. et al., J.Am. Chem. Soc., 99(1977), 3522.

[7] Cheung, S.K. et al., ibid, 98(1976), 5028.

[8] Walker, F.A., ibid, 92(1970), 4235.

[9] Hoffman, B.M. et al., ibid. 98(1976), 5473. 